\title{
Respostas Correlacionadas em Características Reprodutivas no Programa de Melhoramento do Ecótipo Mantiqueira para Produção de Leite ${ }^{1}$
}

\section{Marcos Vinícius Gualberto Barbosa da Silva ${ }^{2,3}$, Jaime Araujo Cobuci ${ }^{3}$, William José Ferreira ${ }^{3}$, Guilherme Paes Guaragna2, Paulo Rogério Palma de Oliveira²}

\begin{abstract}
RESUMO - Por meio de análises bivariadas entre produção total de leite (PL) e idade ao primeiro parto (IDP), ou intervalo de partos (IDP), ou período de serviço (PSERV), objetivou-se com este trabalho estimar parâmetros genéticos, correlações simples e de ordem entre os valores genéticos, bem como as respostas correlacionadas nas características reprodutivas, utilizando-se registros provenientes das cinco primeiras lactações de 1406 vacas do ecótipo Mantiqueira, filhas de 113 reprodutores, com partos entre os anos de 1952 e 1997, pertencentes ao programa de melhoramento do NPZGJRA/IZ/APTA/SAA-SP. Nas análises, foi utilizado o sistema MTDFREML com modelos que incluíam, para as características PL, IDP e PSERV, os efeitos fixos de ano-estação de parto; como covariável, a idade da vaca ao parto, em meses, com termos linear e quadrático, além dos efeitos aleatórios de animal, de ambiente permanente e erro. Nas análises envolvendo a PL, incluiu-se, ainda, o efeito fixo da duração da lactação. No estudo de IPP, o modelo utilizado considerou o efeito fixo de ano-estação de nascimento e, como efeitos aleatórios, animal e erro. As correlações genéticas entre PL e IPP, ou IDP, ou PSERV, foram, respectivamente de, $-0,63,-0,41$ e -0,37. Esses valores evidenciam associações desejáveis entre as características reprodutivas e produção de leite. As respostas correlacionadas, as diretas e a eficiência da seleção indireta foram -0,8904, 1,0165 e -0,8759, para IPP; -3,3520, 2,4651 e -1,3598, para IDP; e -3,0120, 2,4544 e -1,227,2 para PSERV. Esses valores sugerem que a resposta direta para IPP seria mais efetiva que a indireta, ao contrário de IDP e PSERV. As correlações simples e de ordem entre PL e as características reprodutivas foram altas, indicando associação linear e favorável entre os valores genéticos preditos.
\end{abstract}

Palavras-chave: bovino de leite, correlação de ordem, ecótipo Mantiqueira, método REML, modelo animal, resposta correlacionada

\section{Correlated Responses in the Reproductive Traits in the Breeding Program of Mantiqueira Cattle for Milk Production}

\begin{abstract}
The objective of this work was to estimate the genetic parameters, product-moment and rank correlations between breeding values, and also correlated responses for reproductive traits using bivariate analysis of total milk production (PL) and the age at the first calving (IPP), or calving intervals (IDP), or days open (PSERV). Records from the NPZGJRA/IZ/APTA/SAA-SSP breeding program of the first five lactations of 1406 cows of Mantiqueira ecotype; daughters of 113 sires, with calvings from 1952 to 1997 were used. The MTDFREML system was used in the analyses with models for the PL, IDP, and PSERV traits which included the fixed effects of year-season of calving, linear and quadratic terms of calving age as covariate and the random animal effects; environment permanent and error. In the PL analysis, the fixed effect of lactation period was also included. The model for IPP consisted of the year-season fixed effects of calving and the random effects of animal and error. Genetic correlations of PL with IPP, IDP and PSERV were -0.63, -0.41 and -0.37 , respectively, representing favorable associations of milk yield and reproductive traits. Direct, correlated responses and efficiency of the indirect selection were, respectively, $-0.8904,1.0165$ and -0.8759 , for IPP; $-3.3520,2.4561$ and 1.3598 , for IDP and $-3.0120,2.4544$ and -1.2272, for PSERV. These values suggest that the direct response for IPP would be more effective than the indirect, being the opposite for IDP and PSERV. Product-moment and rank correlations of PL with the reproductive traits were high, indicating linear and favorable association between predicted breeding values.
\end{abstract}

Key Words: dairy cattle, rank correlation, Mantiqueira ecotype, REML methodology, animal model, correlated response

\section{Introdução}

O retorno econômico dos sistemas de produção de bovinos leiteiros depende da produção de leite e da eficiência reprodutiva, as quais estão, de certo modo, relacionadas entre si. A produção de leite está sujeita às variações na atividade reprodutiva, pois aos menores intervalos de partos pode estar associada a maior produção de leite na vida produtiva do animal, além do possível aumento no número de animais nascidos por ano. Isto proporcionaria maior rentabilidade econômica, aumento da intensidade seletiva e maiores 
ganhos genéticos. Pelissier (1982), citado por FREEMAN (1984), relatou que, no ano de 1981, os custos totais devidos à baixa fertilidade foram de U\$116.25 por vaca, resultando em uma perda de 1266 bilhões de dólares na pecuária leiteira americana.

Altos níveis de produção de leite e elevados índices de eficiência reprodutiva devem ser, simultaneamente, as metas prioritárias dos produtores. Entretanto, muitas estimativas de correlações genéticas entre características de produção e eficiência reprodutiva, obtidas a partir de análises de dados de campo, sugerem substancial antagonismo entre elas (VAN ARENDONK et al., 1989; SHORT et al., 1990).

Em contraste, em outros estudos foi encontrada pequena ou nenhuma associação da produção de leite com eficiência reprodutiva (DONG e VAN VLECK, 1989 e KAFIDI et al., 1992). Adicionalmente, experimentos delineados de seleção, muitas vezes, não conseguiram demonstrar qualquer antagonismo entre produção e reprodução nas gerações iniciais. Por outro lado, resultados envolvendo várias gerações não estão ainda disponíveis, o que colabora para a incerteza existente a respeito destes possíveis antagonismos.

Em qualquer programa de melhoramento, faz-se necessário o monitoramento da tendência genética ao longo do tempo, com o intuito de se avaliar a mudança ocasionada pelo processo de seleção. Todavia, em vários trabalhos disponíveis na literatura, avaliandose a tendência genética em dados provenientes de rebanhos experimentais ou comerciais, os pesquisadores analisaram basicamente os registros de produção de leite e de seus componentes, não se preocupando com possíveis alterações nas características reprodutivas, em decorrência da seleção direta ou como resposta correlacionada da seleção para as características de produção (ABDALLAH e McDANIEL, 2000).

A importância das características reprodutivas, em programas de melhoramento genético, está relacionada com a taxa de ganho genético anual, pois menores idades ao primeiro parto permitem a redução do intervalo de gerações, enquanto menores períodos de serviço e intervalos de partos disponibilizam maior número de novilhas de alto potencial genético para substituir vacas descartadas (TEIXEIRA et al., 1994).

Assim, por meio de análises bivariadas entre produção de leite e idade ao primeiro parto, ou intervalo de partos, ou período de serviço, objetivou-se com este trabalho estimar parâmetros genéticos, correlações simples e de ordem entre os valores genéticos, bem como as respostas correlacionadas nas características reprodutivas, utilizando-se registros de vacas pertencentes ao programa de melhoramento do ecótipo Mantiqueira do Núcleo de Pesquisa Zootécnica Geraldo José Rodrigues Alckmin do Instituto de Zootecnia da Agência Paulista de Tecnologia dos Agronegócios da Secretaria de Agricultura e Abastecimento do Estado de São Paulo (NPZGJRA/ IZ/APTA/SAA-SP).

\section{Material e Métodos}

Foram utilizados, neste estudo, registros referentes às características produção total de leite (PL), idade ao primeiro parto (IPP), intervalo de partos (IDP) e período de serviço (PSERV), provenientes das cinco primeiras lactações de 1406 vacas do ecótipo Mantiqueira, filhas de 113 reprodutores, com partos entre os anos de 1952 e 1997, obtidas do Arquivo de Escrituração Zootécnica do NPZGJRA/IZ/APTA/SAA-SP.

Este rebanho, formado em 1952, foi incluído em um programa de melhoramento somente a partir de 1977 , com ênfase na seleção para aumento da produção de leite. Procurou-se, então, ter um manejo definido e uniforme, sendo criada a Unidade Experimental de Seleção do Tipo Mantiqueira, cuja principal característica era a predominância do uso de pastagem na alimentação, formada basicamente pelos capins Brachiaria decumbens, Setaria kazungula e Mellinis minutiflora. Na época da seca (abril a setembro), foi fornecida aos animais suplementação volumosa, como silagem de milho ou sorgo. As vacas em lactação foram ordenhadas mecanicamente, sem bezerro ao pé, duas vezes ao dia, recebendo suplementação concentrada, na base de $1 \mathrm{~kg}$ de ração para cada $2,5 \mathrm{~kg}$ de leite produzido, acima de $3 \mathrm{~kg}$. Informações adicionais a respeito da formação do rebanho, manejo adotado e programa de melhoramento foram detalhadas por GUARAGNA et al. (1984).

Para consistência do arquivo analisado, exigiu-se que cada subclasse ano-estação contivesse, no mínimo, 15 observações e que cada reprodutor tivesse filhas em pelo menos dois anos. $\mathrm{O}$ arquivo de pedigree continha 2070 animais, com todas as informações de parentesco disponíveis desde a formação do rebanho em 1952.

Para estimação dos parâmetros genéticos e predição dos valores genéticos, foram realizadas análises bivariadas envolvendo produção de leite e cada uma das características reprodutivas. Utilizou-se 
1230 Rev. bras. zootec.

o sistema MTDFREML (BOLDMAN et al, 1995), com modelos (modelo animal) que incluíam, para as características PL, IDP e PSERV, os efeitos fixos de ano-estação de parto (águas: outubro a março e seca: abril a setembro); como covariável, a idade da vaca ao parto, em meses, com termos linear e quadrático, além dos efeitos aleatórios de animal, de ambiente permanente e erro. Nas análises envolvendo a PL, incluiu-se, ainda, o efeito fixo da duração da lactação. No estudo de IPP, o modelo utilizado considerou o efeito fixo de ano-estação de nascimento e como efeitos aleatórios, animal e o erro.

Além das correlações genéticas aditivas, estimadas pelo método REML, foram obtidas as correlações simples entre os valores genéticos (Pearson) e as correlações de ordem entre as classificações pelos valores genéticos (Spearman), para PL e cada uma das características reprodutivas.

Foram calculadas também as porcentagens de animais em comum, os quais seriam selecionados ou descartados para IPP, ou IDP, ou PSERV, e para PL, para níveis crescentes de seleção ou descarte, respectivamente, de touros e de vacas.

Os ganhos genéticos realizados, para as características estudadas, foram estimados por meio de regressão das médias dos valores genéticos, ponderadas pelo número de observações, em função dos anos de nascimento dos animais.

As respostas da seleção direta para IPP, IDP e PSERV, bem como as respostas correlacionadas para essas características, quando a ênfase na seleção é dada para aumento de produção de leite, foram obtidas pelas fórmulas apresentadas por FALCONER e MACKAY (1996).

A eficiência relativa da seleção indireta e a seleção direta foi obtida pela razão entre a resposta correlacionada para as características reprodutivas quando a seleção foi praticada para produção de leite e o ganho ou resposta direta para as características reprodutivas.

\section{Resultados e Discussão}

\section{Herdabilidades e repetibilidades}

As estimativas das variâncias genética aditiva, de ambiente permanente, residual e fenotípica, bem como de herdabilidade $\left(\hat{h}^{2}\right)$ e repetibilidade $(\hat{r})$, para cada característica estudada, são apresentadas na Tabela 1.

Entre as características ligadas à eficiência reprodutiva, a IPP foi a que apresentou maior $\hat{h}^{2}$. Tal fato também foi observado por PEREIRA (1993), no Brasil, ao analisar registros de IPP em um rebanho da raça Caracu, e por LOPEZ (1986), que encontrou valores entre 0,08 e 0,47 , ao revisar a literatura que trata de estimativas de herdabilidade para IPP em várias raças e cruzamentos de gado leiteiro. $\mathrm{O}$ valor 0,15 , obtido neste trabalho, indica que uma resposta à seleção poderia ser esperada nesta característica, embora melhorias de manejo e nutrição possam constituir maneiras mais rápidas para a redução da sua média.

As $\hat{h}^{2}$ para IDP e PSERV encontradas neste estudo estão em concordância com as normalmente relatadas em trabalhos publicados em países de clima tropical e temperado, as quais são baixas e, muitas vezes, não diferentes de zero, como os descritos, no Brasil, por CARDOSO (1985) e GROSSI e FREITAS (2000), para a raça Holandesa e mestiços HolandêsZebu, respectivamente; e nos Estado Unidos, também para a raça Holandesa, por CAMPOS et al. (1994) e ABDALLAH e McDANIEL (2000). PE-

Tabela 1 - Estimativas de variância genética aditiva $\left(\hat{\sigma}_{\mathrm{a}}^{2}\right)$, de ambiente permanente $\left(\hat{\sigma}_{\mathrm{pe}}^{2}\right)$, residual $\left(\hat{\sigma}_{\mathrm{e}}^{2}\right)$, fenotípica $\left(\hat{\sigma}_{\mathrm{p}}^{2}\right)$, herdabilidade $\left(\hat{\mathrm{h}}^{2}\right)$ e repetibilidade $(\hat{\mathrm{r}})$, para produção de leite $(\mathrm{PL})$, idade ao primeiro parto (IPP), intervalo de partos (IDP) e período de serviço (PSERV)

Table 1 - Estimates of additive genetic variance $\left(\hat{\sigma}_{\mathrm{a}}^{2}\right)$, permanent environment $\left(\hat{\sigma}_{\mathrm{pe}}^{2}\right)$, error $\left(\hat{\sigma}_{\mathrm{e}}^{2}\right)$, phenotypic $\left(\hat{\sigma}_{\mathrm{P}}^{2}\right)$, heritability $\left(\hat{\mathrm{h}}^{2}\right)$ and repeatability ( $\hat{\mathrm{r}})$, for milk yield (PL), age of first calving (IPP) and days open (PSERV)

\begin{tabular}{|c|c|c|c|c|c|c|}
\hline $\begin{array}{l}\text { Características } \\
\text { Characteristics }\end{array}$ & $\hat{\sigma}_{\mathrm{a}}^{2}$ & $\hat{\sigma}_{\mathrm{pe}}^{2}$ & $\hat{\sigma}_{\mathrm{e}}^{2}$ & $\hat{\sigma}_{\mathrm{P}}^{2}$ & $\hat{\mathrm{h}}^{2}$ & $\hat{\mathrm{r}}$ \\
\hline PL1 & 46834,04 & - & 112832,22 & 159666,26 & 0,29 & - \\
\hline PL2 & 74800,47 & 43470,80 & 214893,86 & 333165,13 & 0,22 & 0,35 \\
\hline PL3 & 74757,33 & 43515,40 & 214956,18 & 333228,93 & 0,22 & 0,35 \\
\hline IPP & 6,75 & - & 39,17 & 45,92 & 0,15 & - \\
\hline IDP & 289,63 & 1093,81 & 13807,73 & 15191,21 & 0,02 & 0,09 \\
\hline PSERV & 363,64 & 1009,44 & 13687,68 & 15060,77 & 0,02 & 0,09 \\
\hline
\end{tabular}

\footnotetext{
${ }_{1}$ Análise bivariada entre PL e IPP; ${ }^{2}$ Análise bivariada entre PL e IDP; ${ }^{3}$ Análise bivariada entre PL e PSERV.
}

1 Bivariate analyses of $P L$ and IPP; ${ }^{2}$ Bivariate analysis of $P L$ and IDP; ${ }^{3}$ Bivariate analysis of $P L$ and PSERV. 
REIRA (1993) relatou que estas características são muito influenciadas por fatores de ambiente e genéticos não-aditivos.

Entre as justificativas para os baixos valores de herdabilidade encontrados para IDP e PSERV, pode-se destacar a ação da seleção natural. Provavelmente, a seleção natural tenha alterado a freqüência dos genes ligados à eficiência reprodutiva, com a possível fixação dos genes desejáveis. Além disso, segundo JANSEN (1985), as estimativas de herdabilidade de IDP são obtidas por dados de campo, os quais estão sujeitos à interferência do criador. Por exemplo, as medidas de IDP e PSERV, geralmente, não incluem vacas que foram descartadas por baixa produção ou por problemas reprodutivos, o que certamente diminui a variância genética aditiva destas características. Em contrapartida, se o criador optar por aumentar o PSERV de vacas de alta produção em relação às de baixa produção, a variância genética poderia ser inflacionada. Entretanto, de acordo com a literatura, o efeito do descarte de animais sobre a variância genética parece ser maior que o retardamento do PSERV.

As $\hat{h}^{2}$ para PL obtidas neste estudo situaram-se no intervalo de 0,22 a 0,29 , sendo semelhantes aos valores relatados na literatura por vários autores, em diversas raças e cruzamentos (ALMEIDA et al., 1997; BALIEIRO et al., 1997; GROSSI e FREITAS, 2000). Observa-se que a $\hat{h}^{2}$, obtida na análise bivariada entre PL e IPP, foi maior que as estimativas obtidas nas análises de PL com IDP ou PSERV. Este declínio pode ter sido em função do efeito da seleção dos animais após a primeira lactação, diminuindo a variância genética aditiva. Alguns autores (BAGNATO e OLTENACU, 1994 e SILVA, 1995) revelaram que a utilização de dados selecionados para estimar as (co)variâncias da produção de leite e do intervalo de partos poderia diminuir as estimativas de variâncias genéticas aditivas e residuais, porém a redução observada nas variâncias residuais foi mais acentuada que nas variâncias genéticas aditivas.

As estimativas de repetibilidade encontradas para IDP e PSERV foram iguais a 0,09, sendo próximas às relatadas, na raça Holandesa; por PEREIRA et al. (2000), no Brasil; e por ABDALLAH e McDANIEL (2000), nos Estados Unidos. Contudo, para IDP, esta estimativa é menor que as encontradas por LEMOS (1986), na raça Pitangueiras, e por TEODORO (1991), em animais mestiços, que foram iguais a 0,14 e 0,15 , respectivamente.
Para PL, a $\hat{r}$ obtida $(0,35)$ foi semelhante às encontradas, no Brasil, por LEDIC (1992) e BALIEIRO et al. (1997), em animais da raça Gir e mestiços Holandês-Zebu, respectivamente. Estimativas superiores a essa foram relatadas por RIBAS et al. (1993) e ALMEIDA et al. (1997), ambos em rebanhos da raça Holandesa, no Brasil. Correlações genéticas, fenotípicas e de ambiente

$\mathrm{Na}$ Tabela 2 são apresentadas as correlações genéticas, fenotípicas e de ambiente entre a PL e as características reprodutivas.

A correlação genética entre a PL, na primeira lactação, e a IPP $(-0,63)$ indica que a maioria dos genes que atua sobre a primeira característica também tem efeito, em sentido oposto, sobre a segunda, sugerindo que filhas de touros com alto valor genético para PL tendem a apresentar crescimento mais acelerado ou maturidade fisiológica a uma idade mais precoce (SILVA et al., 1998). Assim, pode-se concluir que a seleção para produção de leite resultaria em novilhas parindo mais cedo, o que está de acordo com os resultados apresentados por SEYKORA e McDANIEL (1983) e RAHEJA et al. (1989). Resultados opostos, no entanto, foram constatados por ABUBAKAR et al. (1987) e REGE (1991). A correlação de ambiente foi inferior e de sinal contrário à correlação genética. Diante desse resultado, denota-se que os animais mais jovens, por ocasião do primeiro parto, tendem a produzir mais leite, devido aos efeitos genéticos aditivos. A correlação fenotípica, por ser superior, é indicativo de que as duas características não são fenotipicamente independentes.

De acordo com SILVA et al. (1998), a magnitude destas correlações é dependente dos valores das herdabilidades para idade ao primeiro parto e produção

Tabela 2 - Correlações genéticas, de ambiente e fenotípicas entre produção de leite $(\mathrm{PL})$, idade ao primeiro parto (IPP), intervalo de partos (IDP) e período de serviço (PSERV)

Table 2 - Genetic, environmental and phenotypic correlations between milk yield (PL), age of first calving (IPP), calving intervals (IDP) and days open (PSERV)

\begin{tabular}{lccc}
\hline & \multicolumn{3}{c}{$\begin{array}{c}\text { Correlações } \\
\text { Correlations }\end{array}$} \\
\cline { 2 - 4 } Características & Genéticas & De ambiente & Fenotípicas \\
Characteristics & Genetic & Environmental & Phenotypic \\
\hline PL-IPP & $-0,63$ & 0,16 & 0,71 \\
PL-IDP & $-0,41$ & 0,00 & 0,43 \\
PL-PSERV & $-0,37$ & 0,00 & 0,43 \\
\hline
\end{tabular}


1232 Rev. bras. zootec.

de leite. Assim, com uma herdabilidade baixa $(0,15)$ e a outra intermediária $(0,29)$, a contribuição da correlação de ambiente para a correlação fenotípica é maior que a contribuição da correlação genética. VAN DAM et al. (1988) não encontraram qualquer relação significativa entre idade ao primeiro parto e produção de leite, mas observaram tendência de animais mais velhos produzirem mais leite que os jovens.

As estimativas obtidas para as correlações genéticas da PL com IDP ou PSERV foram moderadas e com sinais negativos, $-0,41$ e - 0,37 , respectivamente, sugerindo que é possível obter ganhos indiretos para as características reprodutivas por meio da seleção direta para produção de leite. As correlações fenotípicas da PL com IDP ou PSERV foram moderadas, o que pode indicar a existência de dependência fenotípica entre PL e IDP ou PSERV, enquanto as de ambiente foram nulas, revelando que as características não seriam influenciadas pelos mesmos fatores de ambiente. Neste caso, há indícios de que as causas da variação genética e de ambiente influenciam as características por meio de diferentes mecanismos fisiológicos (FALCONER e MACKAY,1996).

\section{Resposta correlacionada}

KRAGELUND et al. (1979) expressaram preocupação em relação às conclusões de alguns trabalhos referentes às associações entre produção de leite e medidas de eficiência reprodutiva, em que foram analisadas, simplesmente, as herdabilidades e correlações de tais características, preferencialmente às possíveis respostas correlacionadas esperadas por seleção. Como neste estudo as herdabilidades para as características reprodutivas foram baixas e as correlações genéticas entre elas e a PL, moderadas, é importante estimar as possíveis mudanças genéticas obtidas por meio de resposta correlacionada. Supondo-se intensidades de seleção iguais para todas as características e seleção direta para PL, as respostas correlacionadas, diretas e a eficiência da seleção indireta foram, respectivamente, $-0,8904,1,0165$ e - 0,8759 , para IPP; -3,3520, 2,4651 e -1,3598, para IDP; e para PSERV de $-3,0120,2,4544$ e $-1,2272$.

Verifica-se que a seleção indireta é menos eficiente que a seleção direta para IPP, provavelmente em razão da existência de variabilidade genética suficiente para proporcionar maiores ganhos diretos. A eficiência da seleção indireta poderia ser maior, caso a correlação entre as características fosse mais elevada e/ou a herdabilidade da IPP fosse menor.

Em relação a IDP e PSERV, pelo fato de suas herdabilidades serem próximas de zero e as correlações genéticas com a PL serem moderadas e com sinais negativos, parece ser interessante proceder à seleção somente para o aumento da PL, pois seriam obtidos maiores ganhos indiretos nessas duas características reprodutivas.

\section{Tendência genética}

As médias das estimativas dos valores genéticos de vacas, por ano de nascimento, para PL e IPP ou IDP, estão apresentadas nas Figuras 1 e 2, respectivamente.

Observa-se que IDP e IPP tiveram comportamentos semelhantes em relação à PL, pois as variações nos valores genéticos médios destas características, quando comparadas com PL, foram antagônicas, o que pode ser explicado pela existência de correlações negativas.

É importante destacar ainda que, em todo o período estudado, o comportamento dos valores genéticos para PSERV foi semelhante ao IDP.

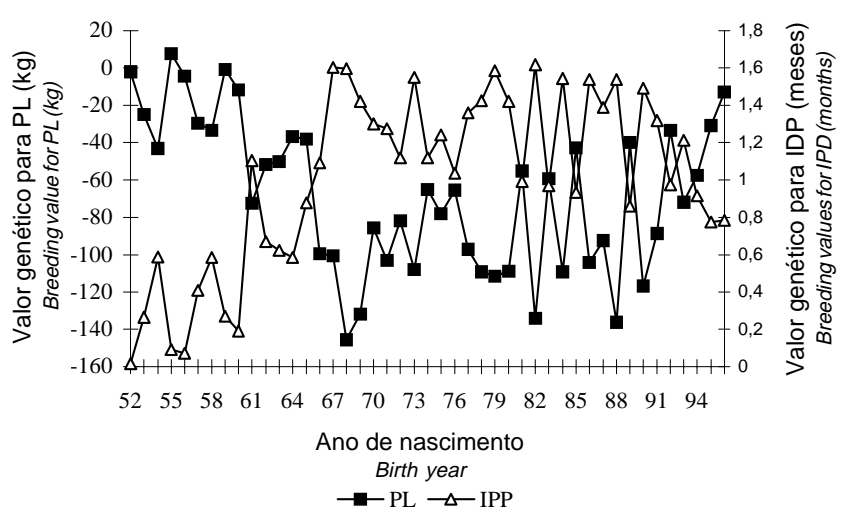

Figura 1 - Valores genéticos médios de vacas, para produção de leite $(\mathrm{PL})$ e idade ao primeiro parto (IPP), de acordo com o ano de nascimento.

Figure 1 - Average cow breeding values for milk yield (PL) and age of first calving (IPP), according to birth year.

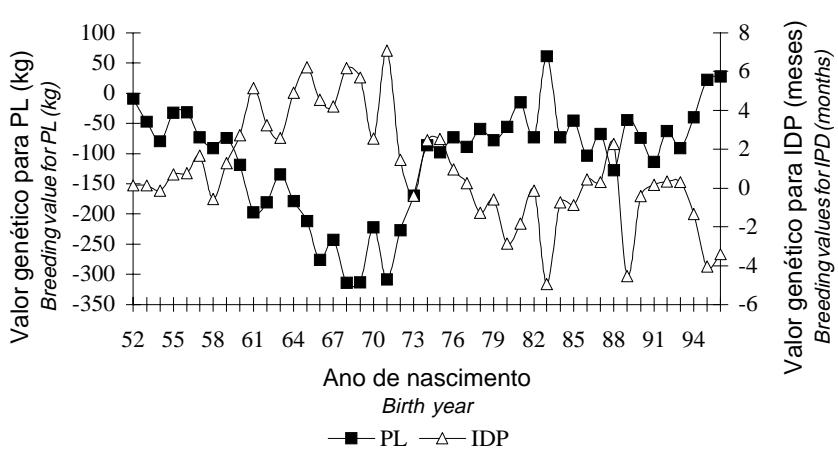

Figura 2 - Valores genéticos médios de vacas, para produção de leite (PL) e intervalo de partos (IDP), de acordo com o ano de nascimento.

Figure 2 - Average cow breeding values for milk yield (PL), calving interval (IDP), according to birth year. 
As mudanças genéticas anuais e respectivos errospadrão para IPP, IDP e PSERV, para touros, foram de $-0,002 \pm 0,01$ meses/ano, $-0,16 \pm 0,05$ dias/anoe $-0,17 \pm 0,06$ dias/ano e para vacas, de $0,005 \pm 0,005$ meses/ano, $-0,13 \pm$ 0,03 dias/anoe $-0,14 \pm 0,03$ dias/ano. Para análise bivariadas entre PL x IPP, PL x IDP e PL x PSERV, os ganhos obtidos na PL, para touros, foram de 1,44 $\pm 0,97 \mathrm{~kg} / \mathrm{ano}, 5,02 \pm 1,60$ $\mathrm{kg} /$ ano e $5,00 \pm 1,59 \mathrm{~kg} /$ ano e para vacas, de $0,29 \pm 0,53 \mathrm{~kg} /$ ano, $3,46 \pm 0,95 \mathrm{~kg} /$ anoe $3,44 \pm 0,95 \mathrm{~kg} / \mathrm{ano}$. Pelas mudanças genéticas anuais para produção de leite, pode-se constatar que, tanto para touros quanto para vacas, ocorreram pequenos ganhos genéticos ao longo dos anos. Pelo fato de a seleção ser praticada com intuito de aumentar a produçãode leite, observa-se que IPP permaneceu inalterada e pequenas mudanças foram observadas em IDP e PSERV. Estes resultados estão de acordo com os obtidos por meio do estudo das respostas direta e correlacionada.

Correlações entre valores genéticos e correlações de ordem

As correlações simples entre valores genéticos
(Pearson) e as correlações de ordem entre as classificações pelos valores genéticos (Spearman) são apresentadas na Tabela 3.

As correlações simples entre PL e as características reprodutivas foram altas e negativas, indicando associação linear favorável entre os valores genéticos preditos.

As correlações de ordem obtidas também foram altas, sugerindo que os indivíduos com os maiores valores genéticos para PL foram também aqueles com menores valores para IPP, IDP e PSERV. Tal fato pode ser constatado nas Figuras 3 e 4, nas quais tem-se a dispersão das classificações dos touros para PL e IPP ou IDP, respectivamente.

Nas Figuras 5, 6 e 7, encontram-se as porcentagens de animaisemcomum, os quais seriam selecionadosou descartados para PL e para IPP, ou IDP ou PSERV, para níveis crescentes de seleção ou descarte, de touros ou vacas.

Observou-se que, a partir da seleção ou descarte de, aproximadamente, $25 \%$ de touros ou vacas, há tendência de aumento da porcentagem de animais em comum.

Tabela 3 - Correlações simples entre valores genéticos (Pearson) e de ordem entre as classificações dos animais pelos valores genéticos (Spearman), para produção de leite (PL), idade ao primeiro parto (IPP), intervalo de partos (IDP) e período de serviço (PSERV)

Table 3 - Product-moments (Pearson's) and rank correlations (Spearman's) between breeding values for milk yield, age of first calving (IPP), calving intervals (IDP) and days open (PSERV)

\begin{tabular}{|c|c|c|c|c|c|}
\hline \multirow[b]{2}{*}{$\begin{array}{l}\text { Características } \\
\text { Characteristics }\end{array}$} & \multicolumn{5}{|c|}{$\begin{array}{l}\text { Correlações } \\
\text { Correlations }\end{array}$} \\
\hline & Pearson $^{1}$ & Spearman $^{1}$ & Pearson $^{2}$ & Spearman $^{2}$ & Pearson $^{3}$ \\
\hline PL - IPP & $-0,86$ & 0,88 & $-0,86$ & 0,86 & $-0,86$ \\
\hline PL - IDP & $-0,77$ & 0,75 & $-0,75$ & 0,70 & $-0,75$ \\
\hline PL-PSERV & $-0,71$ & 0,71 & $-0,68$ & 0,65 & $-0,69$ \\
\hline
\end{tabular}

${ }^{1}$ Correlação considerando apenas os reprodutores; ${ }^{2}$ Correlação considerando apenas as vacas; ${ }^{3}$ Correlação considerando touros e vacas. ${ }_{1}^{1}$ Correlations considering only sires; ${ }^{2}$ Correlations considering only cows; ${ }^{3}$ Correlations considering sires and cows.

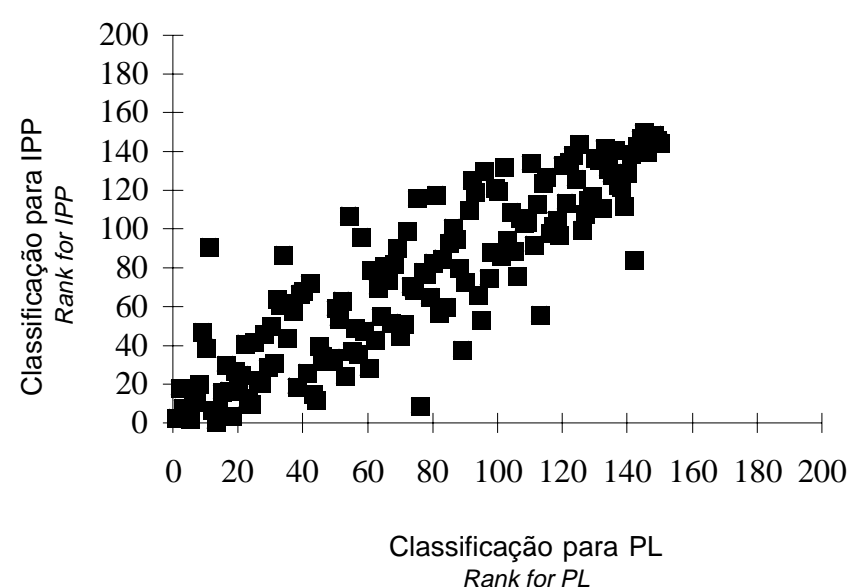

Figura 3 - Dispersão das classificações de touros para produção de leite (PL) e idade ao primeiro parto (IPP).

Figure 3 - Rank dispersions of sires for milk yield (PL) and age of first calving (IPP).

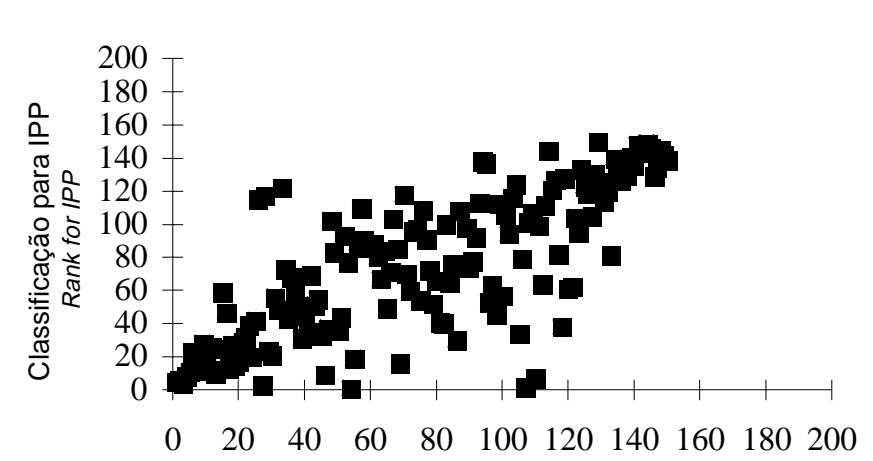

Classificação para PL Rank for $P L$

Figura 4 - Dispersão das classificações de touros para produção de leite $(\mathrm{PL})$ e intervalo de partos (IDP).

Figura 4 - Rank dispersions of sires for milk yield (PL) and calving intervals (IDP). 
1234 Rev. bras. zootec.

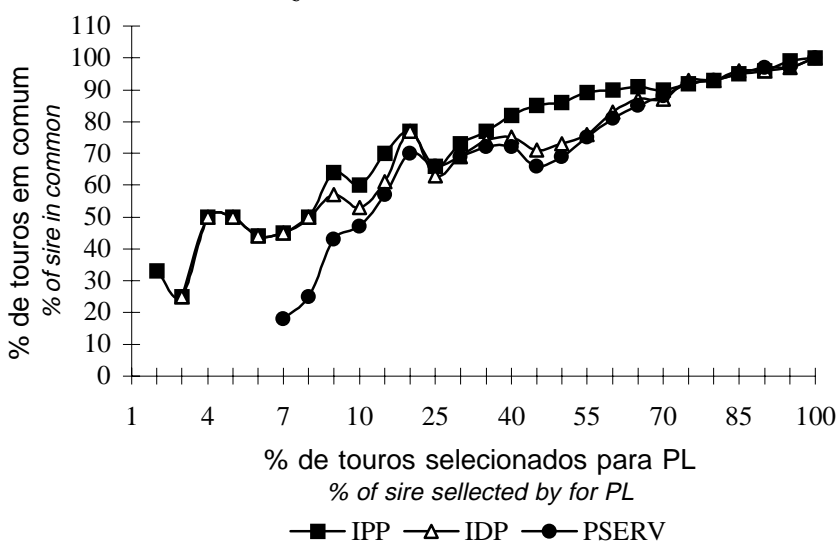

Figura 5 - Porcentagens de touros em comum, selecionados para idade ao primeiro parto (IPP), ou intervalo de partos (IDP), ou período de serviço (PSERV), e para produção de leite $(\mathrm{PL})$, para níveis crescentes de seleção de touros.

Figure 5 - Percentage of sires in common, selected for age of first calving (IPP), or calving intervals (IDP), or days open (PSERV) and milk yield (PL), for increasing levels of sire selection.

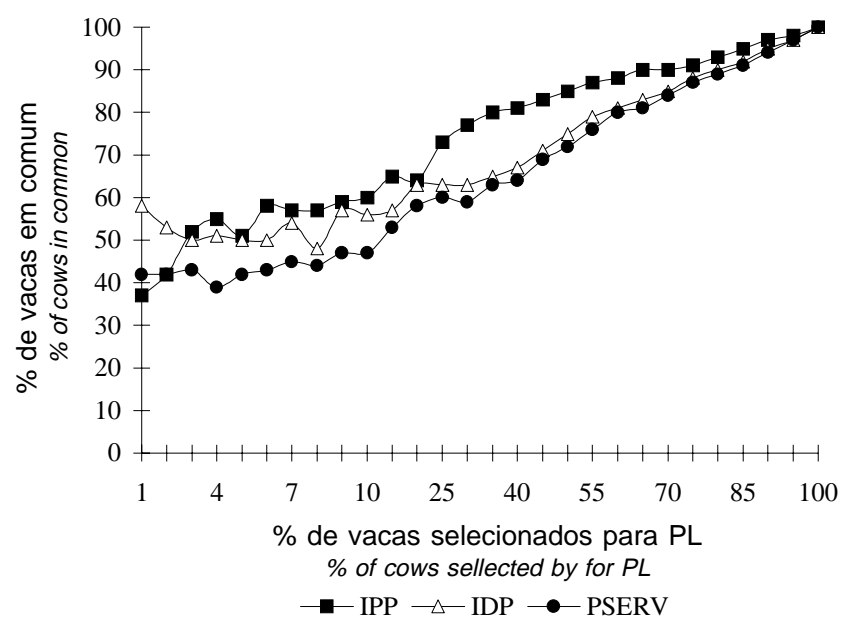

Figura 6 - Porcentagens de vacas em comum, selecionadas para idade ao primeiro parto (IPP), ou intervalo de partos (IDP), ou período de serviço (PSERV), e para produção de leite $(\mathrm{PL})$, para níveis crescentes de seleção de vacas.

Figure 6 - Porcentagens of cows in common, selected for age of first calving (IPP), or calving intervals (IDP), or days open (PSERV) and milk yield (PL), for increasing levels of sire selection.

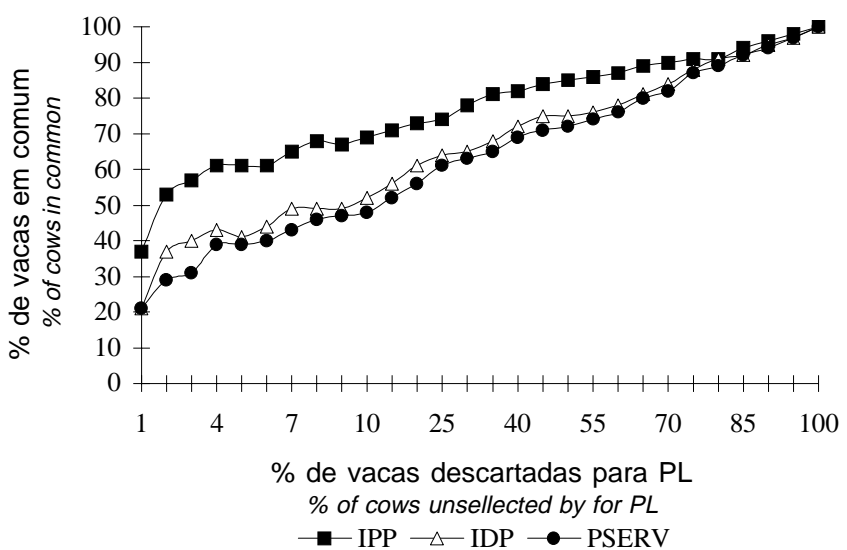

Figura 7 - Porcentagens de vacas em comum, descartadas para idade ao primeiro parto (IPP), ou intervalo de partos (IDP), ou período de serviço (PSERV), e para produção de leite $(\mathrm{PL})$, para níveis crescentes de descarte de vacas.

Figure 7 - Percentage of cows in common, unselected for age of first calving (IPP), or calving intervals (IDP), or days open (PSERV) and milk yield (PL), for increasing levels of sire selection.

\section{Conclusões}

O valor encontrado para a correlação genética evidencia elevado grau de associação entre idade ao primeiro parto e produção de leite e que estas características estão favoravelmente relacionadas.

A seleção direta para diminuição da idade ao primeiro parto é mais eficiente que a seleção indireta, quando a ênfase é para aumento da produção de leite.

Os ganhos genéticos para intervalo de parto e período de serviço podem ser mais efetivos pela resposta correlacionada, quando a seleção é praticada para produção de leite, do que por meio da seleção direta.

De modo análogo às correlações genéticas, as correlações simples e de ordem obtidas sugerem não haver antagonismo entre produção de leite e as características reprodutivas estudadas. Assim, é possível selecionar indivíduos com altos valores genéticos para produção de leite e para idade ao primeiro parto, ou intervalo de partos, ou período de serviço. 


\section{Referências Bibliográficas}

ABDALLAH, J.M., McDANIEL, B.T. 2000. Genetic Parameters and Trends of Milk, Fat, Days Open, and Body Weight After Calving in North Carolina Experimental Herds. J. Dairy Sci., 83(6):1364-1370.

ABUBAKAR, B.Y., McDANIEL, R.E., VAN VLECK, L.D., CABELLO, E. 1987. Phenotypic and genetic parameters for Holsteins in Mexico. Trop. Agricult., 64(1):23-26.

ALMEIDA, R., RIBAS, N. P., MONARDES, H. Estudo dos efeitos genéticos sobre as características produtivas de vacas da raça Holandesa na região da Batavo, Paraná. In: REUNIÃO ANUAL DA SOCIEDADE BRASILEIRA DE ZOOTECNIA, 34, 1997, Juiz de Fora, Minas Gerais. Anais... Juiz de Fora: SBZ, 1997. p.68-70.

BAGNATO, A., OLTENACU, P.A. 1994. Phenotypic evaluation of fertility traits and their association with milk production of Italian Friesian Cattle. J. Dairy Sci., 77(3):874-882.

BALIEIRO, J.C.C., MILAGRES, J.C., FREITAS, A.F. et al. Aspectos Genéticos e Fenotípicos em Características Produtivas do Rebanho Leiteiro da Universidade Federal de Viçosa. In: REUNIÃO ANUAL DA SOCIEDADE BRASILEIRA DE ZOOTECNIA, 34, 1997, Juiz de Fora, Minas Gerais. Anais... Juiz de Fora: SBZ, 1997. p.118-120.

BOLDMAN, K.G., KRIESE, L.A., VAN VLECK, L.D. et al. 1995. A manual for use of MTDFREML. A set of programs to obtain estimates of variances and covariances. [DRAFT]. Beltsville: U.S. Department of Agriculture, Agricultural Research Service. 125p.

CAMPOS, M.S., WILCOX, C.J., BECERRIL, C.M., DIZ, A. 1994 Genetic parameters for yield and reproductive traits of Holsteins and Jersey cattle in Florida. J. Dairy Sci., 77(3):867-873.

CARDOSO, V.L. Efeitos de fatores genéticos e ambientes sobre o desempenho reprodutivo de vacas da raça holandesa. USP, Ribeirão Preto, SP, 1985. 64 p. (Tese de Mestrado).

DONG, M.C., VAN VLECK, L.D. 1989. Estimates of Genetic and Environmental (co)variances for first lactation milk yield, survival, and calving interval. J. Dairy Sci., 72(3):678-684.

FALCONER D.S., MACKAY, T.F.C. 1996. Introduction to Quantitative Genetics. 4.ed. New York: Longman Group Limited. 464p.

FISCHER, L.J., HALL, J.W., JONES, S.E. 1983. Weight and age at calving and weight change related to first lactation milk yield. J. Dairy Sci., 66(10):2167-2172.

FREEMAN A.E. Secondary traits: Sire evaluation and the reproductive Complex. 1984. J.Dairy Sci., 67(2):449-458.

GROSSI, S.F., FREITAS, M.A.R. Fertilidade e produção em rebanhos leiteiros comerciais monitorados por sistema de informação. In: REUNIÃO ANUAL DA SOCIEDADE BRASILEIRA DE ZOOTECNIA, 37, 2000, Viçosa, Minas Gerais. Anais... Viçosa: SBZ, 1997. p. 229. (CDROM).

GUARAGNA, G.P., GAMBINI, L.B., FIGUEIREDO, A.L. 1984. Mantiqueira: 30 Anos Depois. Nova Odessa: Instituto de Zootecnia, Secretaria de Agricultura e Abastecimento de São Paulo. 30p.

JANSEN, J. 1985. Genetic aspects of fertility in dairy cattle based on analysis of A. I. Data - A review with emphasis on areas for further research. Livest. Prod. Sci., 12(1):1-12.

KAFIDI, N., LEROY, P., MICHAUX, C., FRANÇOIS, A. 1992. Relationship between milk production and current calving interval in Belgian Black and White breed. J. Anim. Breed. Genet., 109(2):136-143.

KRAGELUND, K., HILLEL, J., KALAY, D. 1979. Genetic and phenotypic relationship between reproduction and milk production. J. Dairy Sci., 62(3):468-474.

LEDIC, I.L. Investigação sobre produção de leite e peso ao parto em gado Gir. 1992. R. Soc. Bras. Zootec., 21(5):815-826.

LEMOS, A.M. Estudo genético da tolerância ao calor e sua relação com os aspectos reprodutivos e produtivos em bovinos da raça Pitangueiras. Ribeirão Preto, SP: USP, 1986. 113 p. Tese (Doutorado em Melhoramento Animal) Universidade de São Paulo, 1986.

LOPEZ, D. Productive characteristics of bovine cattle in the tropics. II. Reproductive performance. 1986. Cuban. J. Agric. Sci., 20(3):213-222.

PEREIRA, I.G., GONÇALVES, T.M, OLIVEIRA, A.I.G. et al. 2000. Fatores de variação e parâmetros genéticos dos períodos de serviço e seco em bovinos da raça Holandesa no estado de Minas Gerais. Rev. bras. zootec., 29(4):1005-1013.

PEREIRA, J.C.C. Estudo da relação entre características produtivas e reprodutivas de um rebanho bovino nativo da raça Caracú. Belo Horizonte: Escola de Veterinária - UFMG, 1993. 128 p. (Tese de Doutorado).

RAHEJA, K.L., BURNSIDE, E.B., SCHAEFFER, L.R. 1989. Heifer fertility and its relationship with cow fertility and production traits in Holstein dairy cattle. J. Dairy Sci., 72(10):2665-2669.

REGE, J.E.O. 1991. Genetic analysis of Reproductive and Productive performance of Friesian Cattle in Kenya. I. Genetic and phenotipic parameters. J. Anim. Breed. Genet., 108(6):412-423.

RIBAS, N.P., RORATO, P.R.N., LOBO, R.B. 1993. Estimativas de parâmetros genéticos para as características de produção da raça Holandesa no Estado de Paraná. R. Soc. Bras. Zootec., 22(4):634-641.

SEYKORA, A.J. \& McDANIEL, B.T. 1983. Heritabilities and correlations of lactation yields and fertility for Holsteins. J. Dairy Sci., 66(7):1486-1493.

SHORT, T.H., BLAKE, R.W., QUAAS, R.L., VAN VLECK, L.D. 1990. Heterogeneous within-herd variance. 2. Genetic relationship between milk yield and calving interval in grade Holsteins cows. J. Dairy Sci., 73(11):3321-3329.

SILVA, M.V.G.B. Utilização de modelos animais uni e bivariados no estudo das relações entre eficiência reprodutiva e produção de leite na raça Holandesa. Belo Horizonte: Escola de Veterinária - UFMG, 1995. 187 p. (Dissertação de Mestrado).

SILVA, M.V.G.B., BERGMANN, J.A.G., MARTINEZ, M. L. et al. 1998. Associação genética, fenotípica e de ambiente entre medidas de eficiência reprodutiva e produção de leite na raça Holandesa. Rev. bras. zootec., 27(6):1115-1122.

TEIXEIRA, N.M., FREITAS,A.F., RIBAS, N.P. et al. 1994. Tendências genéticas em rebanhos da raça Holandesa no Estado do Paraná. II. Idade ao primeiro parto e primeiro intervalo de partos. R. Soc. Bras. Zootec., 23(6):992-1001

TEODORO, R.L. Estudo comparativo do desempenho de vacas mestiças Holandês: Gir e vacas do cruzamento tríplice Jersey ou Suiço x Holandês:Gir. Viçosa, MG: UFV, 1991. 156p. Tese (Doutorado em Genética e Melhoramento) - Universidade Federal de Viçosa, 1991.

VAN ARENDONK, J.A.M., HOVENIER, R., DE BOER, W. 1989. Phenotypic and genetic association between fertility and production in dairy cows. Livest. Prod. Sci., 21(1):1-12.

VAN DAM, B.P., BARTLETT, P.C., KIRK, J.H., MATHER, E.C. 1988. The effect of age at calving on reproduction, milk production and disease incidence in the first lactation of dairy heifers. Theriogenology, 30(3):583-591.

Recebido em: 26/10/00

Aceito em: 13/03/01 